\title{
Adaptive Fuzzy Segmentation of 3D MR Brain Images
}

\author{
Alan Wee-Chung Liew ${ }^{1}$ and Hong Yan ${ }^{1,2}$ \\ 'Department of Computer Engineering and Information Technology, \\ City University of Hong Kong, \\ 83 Tat Chee Avenue, Kowloon Tong, Hong Kong \\ ${ }^{2}$ School of Electrical and Information Engineering \\ University of Sydney, NSW 2006, Australia \\ Email: \{itwcliew,ityan\}@cityu.edu.hk
}

\begin{abstract}
A fuzzy c-means based adaptive clustering algorithm is proposed for the fuzzy segmentation of 3D MR brain images, which are typically corrupted by noise and intensity non-uniformity (INU) artifact. The proposed algorithm enforces the spatial continuity constraint to account for the spatial correlations between image voxels, resulting in the suppression of noise and classification ambiguity. The INU artifact is compensated for by the introduction of a pseudo-3D bias field, which is modeled as a stack of smooth $B$-spline surfaces with continuity enforced across slices. The efficacy of the proposed algorithm is demonstrated experimentally using both simulated and real MR images.
\end{abstract}

\section{INTRODUCTION}

In 3D MR imaging, inhomogeneity in the magnetic field usually give rises to the so-called Intensity NonUniformity (INU) artifact [1]. This common artifact exhibits itself as a smooth, slowly varying drift in image voxel values across location. Thus, voxels of the same class from different locations may have markedly different feature values. Besides INU artifact, poor contrast between different tissues and severe imaging noise also make accurate segmentation of MR images difficult.

A commonly used image segmentation method is the fuzzy c-means (FCM) clustering algorithm $[2,3]$, which assigns pixels in the image into different classes according to their features. However, plain FCM is clearly inadequate for MR image segmentation due to the following limitations: (i) spatial invariant class features are assumed, i.e., pixels in the same class are assumed to have similar values independent of their locations, (ii) the spatial correlations between data are ignored.

In this paper, we proposed an adaptive FCM algorithm that addresses both the INU artifact and the local spatial correlations (continuity constraint). Our algorithm enforces the continuity constraint using a dissimilarity index in place of the usual distance metric. The INU artifact is compensated for by the introduction of a pseudo-3D spline bias field. Simulation experiment using both simulated and real MRI brain data shows that our algorithm can produce good segmentation results.

\section{PRoBlem Formulation}

The task of 3D MR image segmentation involves the separation of image voxels into regions comprising different tissue types. Let $\underline{x}=(x, y, z)$ be the 3D image coordinate of a voxel. We assume that each tissue class $k$ has a specific feature value $v_{k}$. Then, the ideal signal $o(\underline{x})$ would consist of piecewise constant regions, each having one of the $v_{k}$ values. However, imperfection in the magnetic field often introduces an unwanted low frequency bias term into the signal, which gives rise to the INU artifact. The bias field that gives rise to the INU artifact in an MR image is usually modeled as a smooth multiplicative field [4-7]. The image formation process in MRI can then be modeled as,

$$
s(\underline{x})=o(\underline{x}) b(\underline{x})+n(\underline{x})
$$

where $s(\underline{x})$ is the measured MR signal, $o(\underline{x})$ is the true signal emitted by the tissue, $b(\underline{x})$ is the unknown smoothly varying bias field, and $n(x)$ is an additive noise assumed to be independent of $b(\underline{x})$. Accurate segmentation of an MR image thus involves an accurate estimation of the unknown bias field $b(x)$ and removing this bias field from the measured MR signal. Using the estimated $\tilde{b}(x)$, the log-transformed true signal can be recovered as

$$
\begin{aligned}
\log \tilde{o}(\underline{x}) & =\log s(\underline{x})-\log \tilde{b}(\underline{x}) \\
& \approx \log (o(\underline{x})+n(\underline{x}) / \widetilde{b}(\underline{x}))
\end{aligned}
$$

\section{METHOD}

\section{A. Spatial Continuity Constraint}

The incorporation of local spatial continuity considers the influence of neighboring voxels on the center voxel of interest during classification [8]. Let $\aleph_{\rho}$ denote a chosen 3D local neighborhood configuration with respect to a center voxel $\rho$. Let $\operatorname{dist}(a, b)=\sqrt{\|a-b\|^{2}}$ denote the $L_{2}$ distance between vectors $a$ and $b$. For every voxel $s(\underline{x})$ in the 3D MR image, we compute the following $L_{2}$ distances,

$$
\begin{aligned}
& \partial_{\underline{x}, \underline{y}}=\operatorname{dist}(s(\underline{x}), s(\underline{y})), s(\underline{y}) \in \mathfrak{N}_{\underline{x}} \\
& d_{k, \underline{x}}=\operatorname{dist}\left(s(\underline{x}), v_{k}\right)
\end{aligned}
$$

where $\aleph_{\underline{x}}$ is the neighborhood of $s(\underline{x})$ and $v_{k}$ is the centroid of the $k$-th cluster. If $\partial_{\underline{x}, \underline{x}}$ is small, we would like $d_{k, \underline{x}}$ to be greatly influence by $d_{k, \underline{y}}$. Otherwise, $d_{k, \underline{x}}$ should be largely independent of $d_{k, \underline{y}}$. Taking all voxels in $\kappa_{\underline{x}}$ into account, 
we define a dissimilarity index $D_{k, \underline{x}}$, which measures the dissimilarity between $s(\underline{x})$ and the $k$-th cluster centroid $v_{k}$, as

$$
D_{k, \underline{x}}=\frac{1}{\left|\aleph_{\underline{x}}\right|} \sum_{\underline{y} \in \mathbb{N}_{\underline{x}}}\left[d_{k, \underline{x}}^{2} \lambda_{\underline{x}, \underline{y}}+d_{k, \underline{y}}^{2}\left(1-\lambda_{\underline{x}, \underline{y}}\right)\right]
$$

where $\left|\aleph_{\underline{x}}\right|$ is the cardinality of the neighborhood configuration, and $\lambda\left(\partial_{\underline{x}, \underline{y}}\right)=\lambda_{\underline{x}, \underline{y}}$, which ranges between zero and one, is the weighting factor controlling the degree of influence of the neighboring voxels $s(\underline{y}) \in \aleph_{\underline{x}}$ on the center voxel $s(\underline{x})$, defined by

$$
\lambda(\partial)=\frac{1}{1+e^{-(\partial-\mu) / \sigma}}
$$

The parameters $\mu$ and $\sigma$ specify the displacement of $\lambda$ from zero, and the steepness of the sigmoid curve, respectively.

The parameter $\mu$ can be viewed as measuring the average "randomness" of the homogeneous region with respect to the chosen neighborhood $\mathfrak{N}_{\underline{x}}$. Assuming that the majority of $\mathfrak{N}_{\underline{x}}$ fall on homogeneous regions, the parameter $\mu$ can be computed by

where

$$
\begin{aligned}
& \mu=\frac{1}{|I|} \sum_{\underline{x} \in I} \partial_{a v}(\underline{x}) \\
& \partial_{a v}(x)=\frac{1}{\left|\aleph_{\underline{x}}\right|} \sum_{\underline{y} \in \aleph_{\underline{x}}} \partial_{\underline{x}, \underline{y}}
\end{aligned}
$$

Thus, when the difference between $s(\underline{x})$ and its neighbor $s(\underline{y})$ is much larger than the average "randomness", i.e., $\partial \gg \bar{\mu}$, $s(\underline{x})$ and $s(\underline{y})$ are less likely to belong to the same class, and the influence of $s(\underline{y})$ on the center voxel $s(\underline{x})$ is suppressed in $D_{k, \underline{x}}$.

The steepness parameter $\sigma$ controls the degree of influence of the neighboring voxels on the center voxel and should be chosen such that the clustering results of important image structures are not smoothed out. $\sigma$ could be determined as follows. From the $\partial_{a v}(x)$ computed over the image data, we take $\partial$, equal to the 95 percentile of $\partial_{a v}(x)$. Then we let $\lambda\left(\partial_{t}\right)=0.8$ and solve for $\sigma$ using (6).

The dissimilarity index $D_{k, x}$ effectively smoothes the cluster assignment of $s(\underline{x})$ by the cluster assignment of its neighboring voxels. It enables spatial interaction between neighboring voxels and is adaptive to image content.

The spatial continuity constraint also has a noise suppression capability due to the adaptive smoothing operation. Random noise would perturb the distance of the center voxel and the distances of its neighbors to the cluster centroids. When the weighted average of these distances, i.e., $(5)$, is taken, the effect of random noise is smoothed out.

\section{B. INU Bias Field Compensation}

When a bias field is present, the piecewise constant assumption of MRI data is no longer valid. In view of the MR image formation model of (1), the data should be compensated for the bias field when computing the $L_{2}$ distance between the data and the cluster centroids, i.e., $d_{k, \underline{x}}^{2}$ should be given by

$$
d_{k, \underline{x}}^{2}=\left\|s(\underline{x}) / \tilde{b}(\underline{x})-v_{k}\right\|^{2}
$$

where $\tilde{b}(\underline{x})$ is the estimate for the unknown bias field $b(\underline{x})$.

Instead of estimating the bias field $\tilde{b}(\underline{x})$ in (9) directly, we estimate its $\log$-transformation. Let $w(\underline{x})=\log \tilde{b}(\underline{x})$. For computational consideration, we model the $3 \mathrm{D}$ log bias field $w(\underline{x})$ as a stack of $2 \mathrm{D}$ spline surfaces $\left\{w_{z}(x, y)\right\}$, where each of the spline surfaces $w_{z}(x, y)$ is computed over the $2 \mathrm{D} x-y$ plane at the particular $z$ index. The 2D surfaces are then coupled together to form a smooth $3 \mathrm{D}$ field.

Specifically, we consider the cubic $B$-spline [9], which has a continuous derivative up to the second order. The normalized cubic $B$-spline basis $N_{i, 4}$ with knots $\lambda_{i}, \ldots, \lambda_{i+4}$ is given by

$$
N_{i, 4}(x)=\left(\lambda_{i+4}-\lambda_{i}\right) \sum_{j=0}^{4} \frac{\left(\lambda_{i+j}-x\right)_{+}^{3}}{\prod_{\substack{l=0 \\ l \neq j}}^{4}\left(\lambda_{i+j}-\lambda_{i+1}\right)}
$$

where

$$
\left(\lambda_{i+j}-x\right)_{+}^{3}=\left\{\begin{array}{cl}
\left(\lambda_{i+j}-x\right)^{3}, & \text { if } \lambda_{i+j} \geq x \\
0, & \text { otherwise }
\end{array}\right.
$$

The 2D $\log$ bias field $w_{z}(x, y)$ at index $z$ is formed by the tensor products of cubic $B$-spline bases, i.e.,

$$
w_{z}(x, y)=\sum_{i=-3}^{g} \sum_{j=-3}^{h} \alpha_{i j}^{z} N_{i, 4}(x) M_{j, 4}(y)
$$

with the knot sequences $\left\{\lambda_{-3}, \hat{\lambda}_{-2}, \ldots, \lambda_{g}\right\}$ and $\left\{\mu_{-3}, \mu_{-2}, \ldots, \mu_{h}\right\}$. The superscript $z$ on the spline coefficients $\left\{\alpha_{i j}^{z}\right\}$ denotes that they are for the spline surface at index $z$. Using the tensor product spline representation of $w_{z}(x, y)$, the computation of the $\log$ bias field becomes that of finding the set of $B$-spline coefficients $\left\{\alpha_{i j}^{z}\right\}$.

\section{The Proposed Algorithm}

The objective function for the proposed adaptive spatial FCM (ASFCM) clustering algorithm is given by,

$$
J_{A S F C M}=\sum_{\underline{x} \in I} \sum_{k=1}^{c} u_{k, \underline{x}}^{m} D_{k, \underline{x}}+\beta \eta\left(w_{z}(x, y)\right)+\gamma \varphi\left(w_{z}(x, y)\right)
$$

with $D_{k, \underline{x}}$ defined by (5) and (9), subject to

$$
\sum_{k=1}^{c} u_{k, \underline{x}}=1 \quad \forall \underline{x} \in I
$$

The first regularizing term is given by 


$$
\begin{aligned}
\eta\left(w_{z}(x, y)\right)= & \iint\left\{\left[\frac{\partial^{2} w_{z}(x, y)}{\partial x^{2}}\right]^{2}+2\left[\frac{\partial^{2} w_{z}(x, y)}{\partial x \partial y}\right]^{2}\right. \\
& \left.+\left[\frac{\partial^{2} w_{z}(x, y)}{\partial y^{2}}\right]^{2}\right\} d x d y
\end{aligned}
$$

and the second regularizing term is given by

$$
\varphi\left(w_{z}(x, y)\right)=\iint\left[\frac{\partial^{2} w_{z}(x, y)}{\partial z^{2}}\right]^{2} d x d y
$$

The first regularizing term minimizes the thin plate energy of each of the spline surfaces $w_{z}(x, y)$. The second regularizing term forces smoothness between slices of spline surfaces. It couples the slices together to form a smooth $3 \mathrm{D}$ field. The parameters $\beta$ and $\gamma$ control the fidelity of the fit to the data and the smoothness of the field.

The necessary conditions for the minimization of $J_{A S F C M}$ over the memberships $u_{k, x}$, cluster centroids $v_{k}$, and Bspline coefficients $\alpha_{i j}^{z}$ are obtained by setting the respective first partial derivatives of $J_{A S F C M}$ to zero while keeping the other variables constant.

Consider the following Lagrange functional,

$$
L(u)=J_{A S F C M}(u)+\sum_{\underline{x} \in I} \delta_{\underline{x}}\left(1-\sum_{k=1}^{c} u_{k, \underline{x}}\right)
$$

where $\delta$ is the Lagrange multiplier. Differentiating $L$ with respect to $u_{k, \underline{x}}$, setting the result to zero and using (14) yields,

$$
u_{k, \underline{x}}=\left[\sum_{l=1}^{c}\left(\frac{D_{k, \underline{x}}}{D_{l, \underline{x}}}\right)^{1 / m-1}\right]^{-1}
$$

Differentiating $J_{A S F C M}$ with respect to $v_{k}$ and setting the result to zero yields,

$$
v_{k}=\frac{\sum_{\underline{x} \in I} u_{k, \underline{x}}^{m} g_{\underline{x}}}{\sum_{\underline{x} \in I} u_{k, \underline{x}}^{m}}
$$

where

$$
g_{\underline{x}}=\frac{1}{\left|\hat{N}_{\underline{x}}\right|} \sum_{\underline{y} \in \mathbb{N}_{\underline{x}}}\left[\lambda_{\underline{x}, \underline{y}}(s(\underline{x}) / \tilde{b}(\underline{x}))+\left(1-\lambda_{\underline{x}, \underline{y}}\right)(s(\underline{y}) / \tilde{b}(\underline{y}))\right]
$$

In deriving the first derivative of $J_{A S F C M}$ with respect to the $B$-spline coefficients $\left\{\alpha_{i j}^{2}\right\}$, we ignore the spatial interactions between neighboring voxels. Since the bias field is estimated in the log domain, the original $d_{k, \underline{x}}^{2}$ of (9) is replaced by the following expression

$$
d_{k, \underline{x}}^{2}=\left\|\hat{s}(\underline{x})-w(\underline{x})-\hat{v}_{k}\right\|^{2}
$$

where $w(\underline{x})$ is the $\log$ bias field, $\hat{s}(\underline{x})=\log s(\underline{x})$ and $\hat{v}_{k}=\log v_{k}$.

To find an expression for $\left\{\alpha_{i j}^{z}\right\}$, we fix $u_{k, \underline{x}}$ and $v_{k}$, and discretize the second regularizing term using finite difference, i.e.,

$$
\iint\left[\frac{\partial^{2} w_{z}}{\partial z^{2}}\right]^{2} d x d y=\iint\left[w_{z-2}-4 w_{z-1}^{\prime}+6 w_{z}-4 w_{z+1}+w_{z+2}\right]^{2} d x d y
$$

Then, differentiating the modified $J_{A S F C M}$ with respect to $\alpha_{p q}^{z}$ for a particular $w_{z}(x, y)$, and setting the result to zero, yields the following set of linear equations,

$$
\begin{aligned}
& \left\{\sum_{i=-3}^{g} \sum_{j=-3}^{h} \alpha_{i j}^{z}\left(L_{z}(p, q, i, j)-b_{p, q}^{z}\right\}\right. \\
& \quad+\left\{\sum _ { i = - 3 } ^ { g } \sum _ { j = - 3 } ^ { h } \left[\beta \alpha_{i j}^{z}\left(\omega_{1}(p, q, i, j)+2 \omega_{2}(p, q, i, j)\right)\right.\right. \\
& \left.+\omega_{3}(p, q, i, j)+\gamma D_{z}\left(\alpha_{i, j}\right) \omega_{0}(p, q, i, j)\right]=0
\end{aligned}
$$

for all $p=-3, \ldots, g$ and $q=-3, \ldots h$, where

$$
L_{z}(p, q, i, j)=\sum_{(x, y) \in I} \sum_{k=1}^{c} u_{k, \underline{x}}^{m} N_{p, 4}(x) M_{q, 4}(y) N_{i, 4}(x) M_{j, 4}(y)
$$

$b_{p, q}^{z}=\sum_{(x, y) \in I} \sum_{k=1}^{c} u_{k, \underline{x}}^{m}\left(\hat{s}(\underline{x})-\hat{v}_{k}\right) N_{p, 4}(x) M_{q, 4}(y)$

$D_{z}\left(\alpha_{i, j}\right)=\alpha_{i j}^{z-2}-4 \alpha_{i j}^{z-1}+6 \alpha_{i j}^{z}-4 \alpha_{i j}^{z+1}+\alpha_{i j}^{z+2}$

$\omega_{0}(p, q, i, j)=\int N_{i, 4}(x) N_{p, 4}(x) d x \int M_{j, 4}(y) M_{q, 4}(y) d y$

$\omega_{1}(p, q, i, j)=\int N_{i, 4}^{\prime \prime}(x) N_{p, 4}^{\prime \prime}(x) d x \int M_{j, 4}(y) M_{q, 4}(y) d y$

$\omega_{2}(p, q, i, j)=\int N_{i, 4}^{\prime}(x) N_{p, 4}^{\prime}(x) d x \int M_{j, 4}^{\prime}(y) M_{q, 4}^{\prime}(y) d y$

$\omega_{3}(p, q, i, j)=\int N_{i, 4}(x) N_{p, 4}(x) d x \int M_{j, 4}^{\prime \prime}(y) M_{q, 4}^{\prime \prime}(y) d y$

where the single and double prime in $N$ and $M$ denote first and second derivatives, respectively. The first curly bracket in (23) corresponds to the data term, whereas the second curly bracket corresponds to the regularizing terms.

Equation (23) indicates that we are trying to fit a smooth spline surface $w_{z}(x, y)$ to the $3 \mathrm{D}$ residual signal between the actual data and a piecewise constant FCM solution at a particular $z$ index. Since the residual is obtained by a 3D FCM-based algorithm, it would inherit the within-slice and between-slice continuity from the 3D data. Fitting slice-wise 2D splines over the residual would therefore not incur significant discontinuity between slices, even without the second regularizing term of (16). Nevertheless, (16) explicitly forces the stack of spline surfaces to be smooth over the $z$ direction. 
To compute the spline coefficients $\left\{\alpha_{i j}^{z}\right\}$, we use a novel two-stage algorithm. In the first stage, the second regularizing term is ignored. The bias field is estimated slice by slice, with no explicit coupling between adjacent slices of spline surface. In the second stage, an iterative procedure is used, whereby the previously computed $\left\{\alpha_{i j}^{z}\right\}$ is updated iteratively, taking into account the explicit coupling between adjacent spline surfaces resulting from the second regularizing term.

\section{MRI'BRAIN IMAGE SEGMENTATION}

The algorithm is tested on both simulated 3D MR images obtained from the BrainWeb Simulated Brain Database at the McConnell Brain Imaging Centre of the Montreal Neurological Institute (MNI), McGill University [10-12], and on real MRI data. Fig. 1 shows a slice of the simulated MRI brain data. The brain image of Fig. 1(a) was generated based on a discrete anatomical normal brain model, and serves as the true model. The image of Fig.1(b) was simulated from the true model with the following settings: $T$, modality, ICBM protocol, slice thickness of $1 \mathrm{~mm}\left(1 \mathrm{~mm}^{3}\right.$ voxels), $3 \%$ noise level and $40 \%$ INU. Fig.1(c) shows the actual bias field that produces the INU artifact.
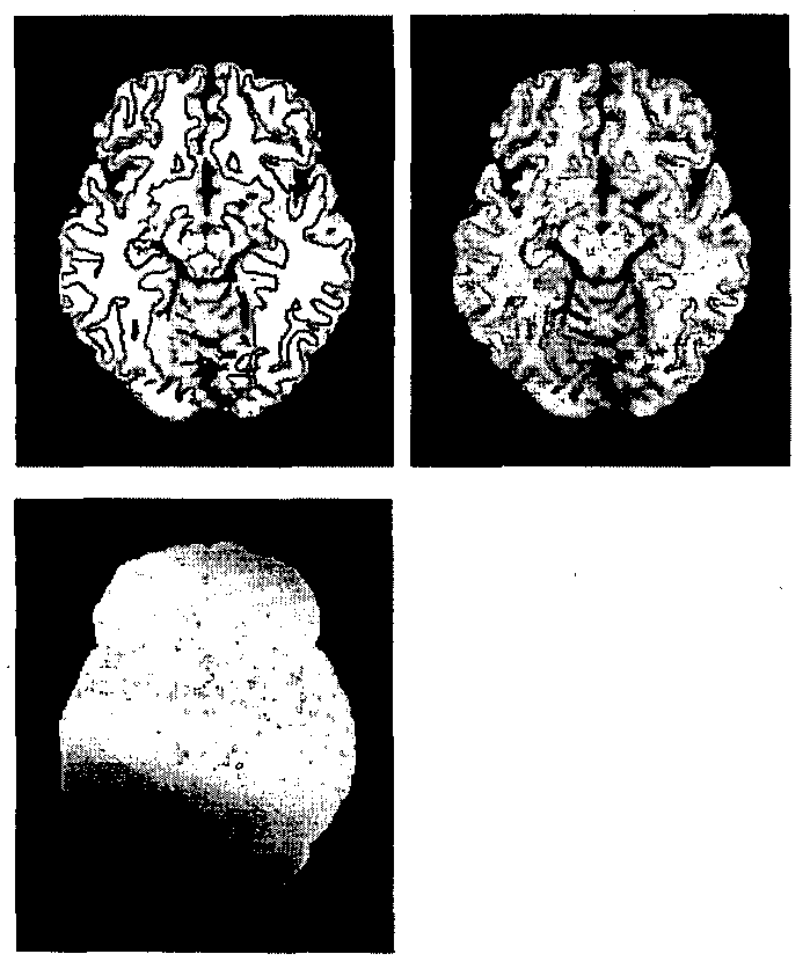

Fig.1 A slice of the simulated 3D brain image from MNI. (a) True model. (b) Image corrupted with noise and INU artifact. (c) The corresponding bias field.
The number of tissue classes in the segmentation was set to three, which corresponds to gray matter (GM), white matter (WM) and cerebrospinal fluid (CSF). Background pixels are ignored in the computation. In all segmentation experiments, the default parameter values are: $m=2, \beta=5000$, number of spline intervals in the $x$ and $y$ dimensions, $n x=n y=2$, maximum number of iterations ITMAX $=50$. A 3D six-point neighborhood is used. The algorithm usually converges in around 6 to 7 iterations. For the simulated 3D MRI brain image of dimension $217 \times 181 \times 181$ (row $(y) \times$ column $(x) \times$ depth $(z)$ ), the total computation time is around 1.5 minutes on a Pentium IV $2 \mathrm{GHz}$ PC.
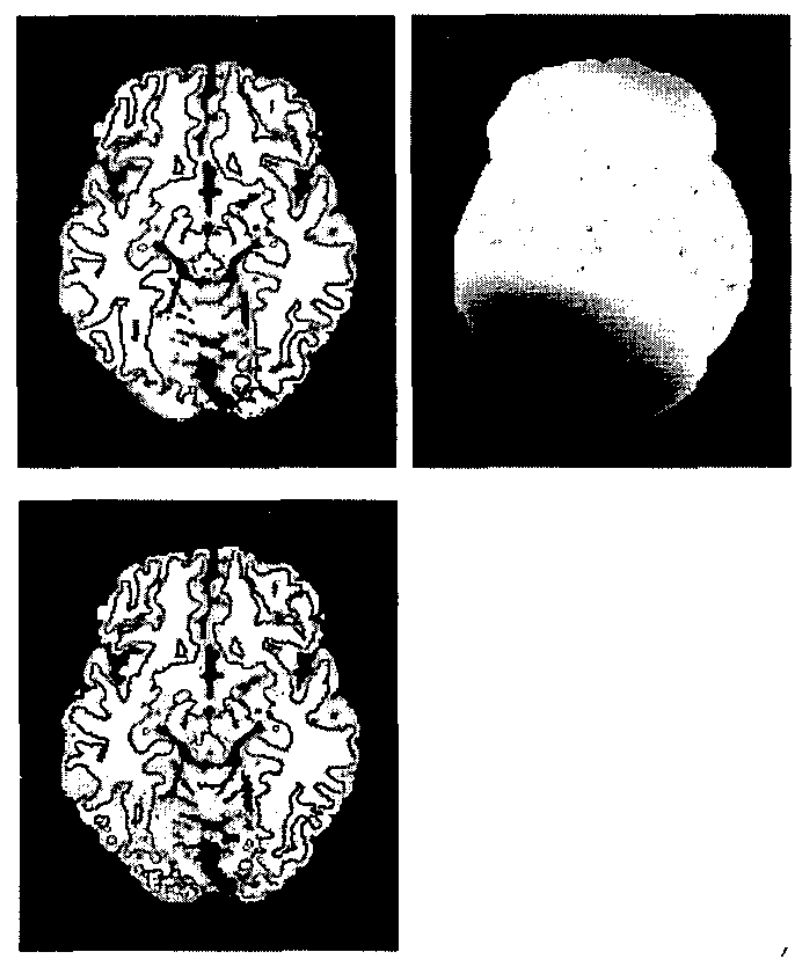

Fig.2 Segmentation result for the MRI image of Fig.1(b). (a) The segmentation using the proposed algorithm, (b) the recovered bias field, (c) the segmentation using the conventional FCM algorithm.
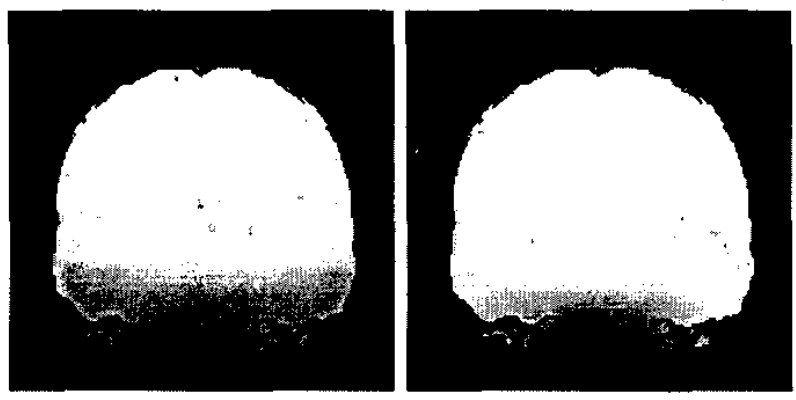

Fig.3 (a) Actual, and (b) computed bias ficld for $y=110$. The $x$ coordinatc increases from left to right and the $z$ coordinate increases from bottom to top. 
Fig.2(a) shows the segmented image. The segmentation can be observed to correspond well to the true model in Fig. 1(a). Fig.2(b) shows the recovered bias field, which resembles very closely the actual bias field of Fig.1(c). In comparison, we also show in Fig.2(c) the segmentation by the conventional FCM algorithm, whose accuracy is severely affected by noise and INU. The results clearly indicate that the proposed algorithm is able to compensate for noise and INU artifact in the input image.

Fig.3(a) shows an across-slice view of the actual bias field from the same data set. Fig.3(b) shows the corresponding estimated bias field. As can be seen, the estimated bias field has captured accurately the intensity inhomogeneity across slices without exhibiting between-slice discontinuity in spite of the modeling of the $3 \mathrm{D}$ bias field by a stack of $2 \mathrm{D}$ spline surfaces. The second regularizing term of (16) has successfully constrained the estimated $3 \mathrm{D}$ field to be smooth in the $z$ direction. To show the effectiveness of the bias compensation method, Fig. 4 shows the mean intensity value of the WM from the base of the brain to the top of the brain. The uncompensated data with $40 \%$ INU (dashed line) shows significant variation in intensity value, while the INU compensated data (solid line) has a more uniform WM intensity value. For comparison, the WM mean intensity value for the data with no INU (dotted line) is also shown. Besides a constant offset, the remarkable similarity in the shape of the two curves indicates that INU has been correctly compensated for. Note that the mean intensity value for the data with no INU is not a constant straight line. This is due largely to partial volume effect, where a voxel is partially shared by two or more tissue types. This phenomenon is particularly noticeable at the two extremes of the brain, where boundaries between tissue types become less defined. The proposed algorithm is also able to correctly take that into account as reflected in the closely matched shape around the two ends of the curve.

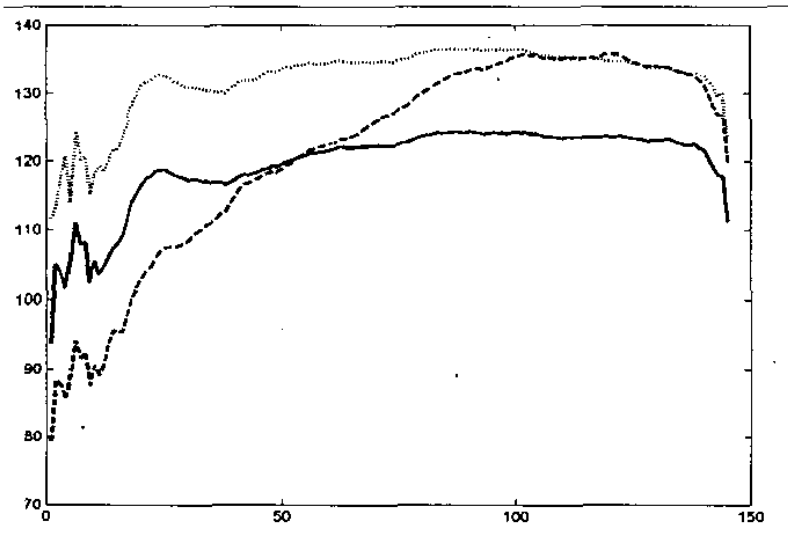

Fig.4 Mean intensity value of WM as a function of $z$-coordinate, from the base of the brain to the top of the brain. The dashed line is for the uncorrected data ( $\mathrm{INU}=40 \%$ ); the solid line is for the INU corrected data. The dotted line is for the data with no INU.
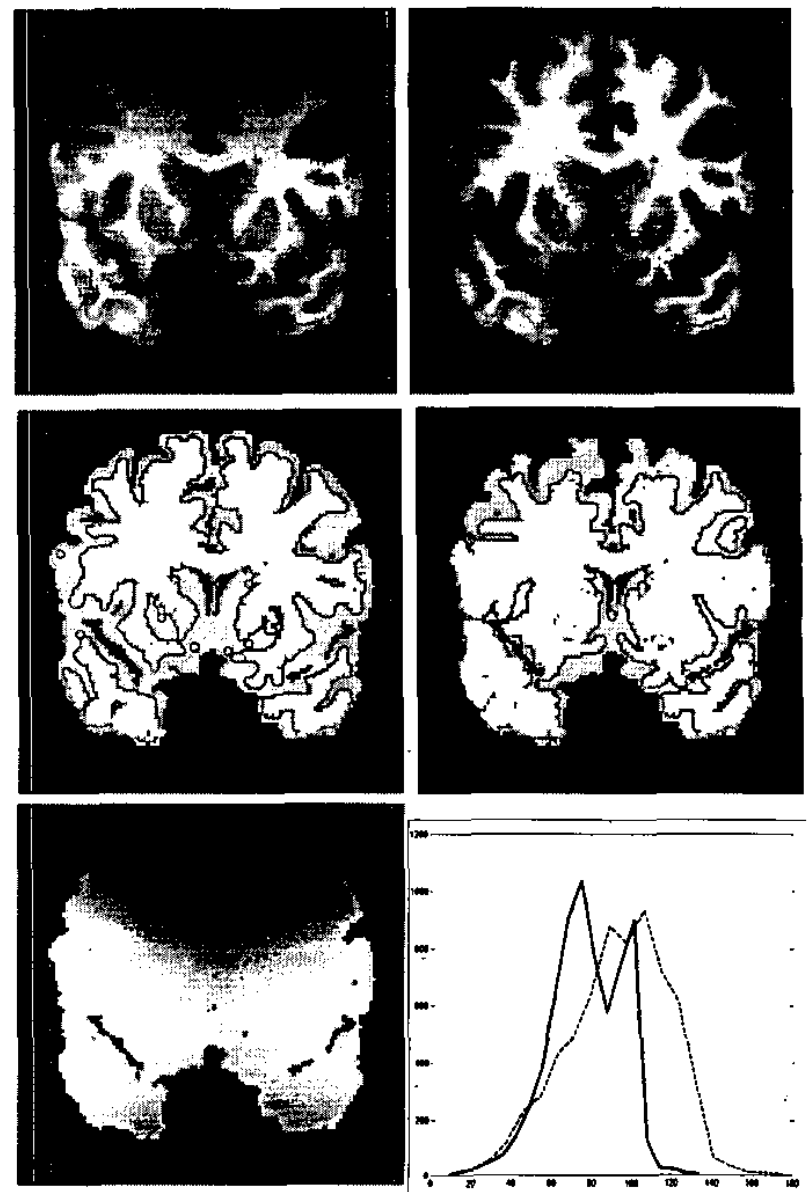

Fig.5 Segmentation of real MRI image. (a) original image, (b) INU corrected image, (c) proposed ASFCM segmentation, (d) FCM segmentation, (e) estimated bias field, ( $f$ intensity histogram before (dashed line) and after (solid line) INU correction.

Fig. 5 shows one slice of the segmentation result for a real $T_{1}$-weighted 3D MR image using the proposed algorithm. Fig.5(a) and 5(b) show the original image and the INU compensated image, respectively. The INU artifact has largely been suppressed and one can see a fairly uniform image compared to the original image. Fig.5(c) and 5(d) show the final segmentation using our method and the FCM algorithm, respectively. Visual inspection shows that our method produces better segmentations than the FCM algorithm. In particular, the GM and WM in the top region of both images can be better resolved by our method. Fig.5(e) shows the estimated bias fields. The bias field has successfully captured the different shading in the original image. Fig.5(f) shows the intensity histogram of the slices before and after INU correction. One can see that there is less spread in the histogram after INU compensation. Also, the two prominent peaks, which correspond to the GM and WM are sharper and better resolved in the $\mathrm{NU}$ compensated image. 


\section{CONCLUSIONS}

We have presented an adaptive spatial fuzzy c-means segmentation algorithm that takes into account local spatial continuity constraint, as well as the suppression of the INU artifact in 3D MR images. The algorithm employs a novel dissimilarity index that considers the local influence of neighboring pixels in an adaptive manner. To suppress the INU artifact, a multiplicative MR image formation model is used. The estimation of the $3 \mathrm{D}$ bias field is then formulated as the estimation of a stack of $2 \mathrm{D}$ smooth spline surfaces with continuity enforced across slices, that minimizes the 3D residual signal between the actual data and a piecewise constant FCM solution. Experimental results on the segmentation of both simulated and real MR brain images illustrate the effectiveness and robustness of our algorithm

\section{ACKNOWLEDGMENT}

This work is supported by the Hong Kong Research Grant Council (Project CityU 1088/00E).

\section{REFERENCES}

[1] A. Simmons, P.S. Tofts, G.J. Barker and S.R. Arridge, "Sources of intensity nonuniformity in spin echo images at 1.5T", Magnetic Resonance Medicine, vol. 32, pp.121$128,1994$.

[2] J.C. Bezdek, L.O. Hall and L.P. Clark, "Review of MR image segmentation techniques using pattern recognition", Medical Physics, vol.20, no. 4, pp.10331048, 1993.

[3] L.O. Hall, A.M. Bensaid, L.P. Clarke, R.P. Velthuizen, M.S. Silbiger and J.C. Bezdek, "A comparison of neural network and fuzzy clustering techniques in segmenting magnetic resonance images of the brain", IEEE Trans. Neural Networks, vol. 3, no. 5, pp. 672-682, September 1992.
[4] C.R. Meyer, P.H. Bland and J. Pipe, "Retrospective correction of intensity inhomogeneities in MRI", IEEE Trans. Medical Imaging, vol. 12, no. 1, pp.36-41, March 1995.

[5] W.M. Wells III, W.E.L. Grimson, R. Kikinis and F.A. Jolesz, "Adaptive segmentation of MRI data", IEEE Trans. Medical Imaging, vol. 15, no. 4, pp.429-442, August 1996.

[6] M. Styner, C. Brechbuhler, G. Szekely and G. Gerig, "Parametric estimate of intensity inhomogeneities applied to MRI", IEEE Trans. Medical Imaging, vol. 9, no. 3, pp.153-165, March 2000.

[7] M. Tincher, C.R. Meyer, R. Gupta and D.M. Williams, "Polynomial modeling and reduction of RF body coil spatial inhomogeneity in MRI", IEEE Trans. Medical Imaging, vol. 12, no. 2, pp. 361-365, June 1993.

[8] A.W.C. Liew, S.H. Leung and W.H. Lau, "Fuzzy image clustering . incorporating spatial continuity", IEE Proceedings-Vision, Image and Signal Processing, vol. 147, no. 2, pp.185-192, April 2000.

[9] P. Dierckx, "Curve and surface fitting with splines", Monographs on Numerical Analysis, Oxford Science Publications, Oxford University Press, Inc., 1993.

[10] http://www.bic.mni.mcgill.ca/brainweb

[11] C.A. Cocosco, V. Kollokian, R.K.S. Kwan and A.C. Evans, "BrainWeb: online interface to a 3D MRI simulated brain database", NeuroImage, vol.5, no.4, part $2 / 4$, S425, 1997, Proceedings of 3rd International Conference on Functional Mapping of the Human Brain, Copenhagen, May 1997.

[12]D.L. Collins, A.P. Zijdenbos, V. Kollokian, J.G. Sled N.J. Kabani, C.J. Holmes and A.C. Evans, "Design and construction of a realistic digital brain phantom", IEEE Trans. Medical Imaging, vol. 17, no. 3, pp. 463-468, June 1998. 